\title{
Augmenting Photosynthesis through Facile AlEgen-Chloroplast Conjugation and Efficient Solar Energy Utilization
}

\author{
Haotian Bai ${ }^{1 \dagger}$, Haixiang Liu, ${ }^{1,4}$, Xu Chen ${ }^{3 \dagger}$, Rong Hu' ${ }^{2}$, Meng Li', Wei He ${ }^{1,4}$, Jian Du', Anjun Qin ${ }^{3}$, Jacky W. Y. \\ Lam $^{1,3}$, Zhiyang Liu ${ }^{1,2^{*}}$, Ryan T. K. Kwok ${ }^{1,4^{*}}$ and Ben Zhong Tang ${ }^{1,3,4^{*}}$
}

\begin{abstract}
${ }^{1}$ Department of Chemical and Biological Engineering, Department of Chemistry, Hong Kong Branch of Chinese National Engineering Research Center for Tissue Restoration and Reconstruction, Division of Life Science and Institute of Advanced Study, The Hong Kong University of Science and Technology, Clear Water Bay, Kowloon, Hong Kong, China.

${ }^{2}$ School of Chemistry and Chemical Engineering, Institute of Advanced Materials, Southeast University, 211189 Nanjing, China.

${ }^{3}$ SCUT-HKUST Joint Research Laboratory, State Key Laboratory of Luminescent Materials and Devices, Guangdong Provincial Key Laboratory of Luminescence from Molecular Aggregates, Center for Aggregation-Induced Emission, South China University of Technology, Guangzhou 510640, China.

${ }^{4}$ HKUST-Shenzhen Research Institute, No. 9 Yuexing 1st RD, South Area, Hi-tech Park Nanshan, Shenzhen 518057, China.

*Correspondence to: Email: liuzhiyang@ seu.edu.cn (Z.L.); chryan@ust.hk (R.T.K.K.); tangbenz@ust.hk (B.Z.T.)
\end{abstract}

$\dagger$ These authors contributed equally to this work.

Photosynthesis is regarded as the foundation for sustaining planet living, and light-harvesting is the initial step of photosystems and activates the subsequent photochemical reactions. However, the incomplete match between the solar radiation spectrum and absorption profile of chloroplasts limits the full absorption and utilization of sunlight by the photosynthetic pigments. Here, we designed two new aggregation-induced emission (AIE)-active molecules with activated alkyl groups (TPE-PPO and TPA-TPO), and realized the substantial manipulation of live chloroplasts via facile metal-free "Click" reaction. Owing to the matched photophysical properties, the AIE luminogens (AIEgens) could harvest harmful ultraviolet radiation (HUVR) and photosynthetically inefficient radiation (PIR), and further convert them into photosynthetically active radiation (PAR) for chloroplasts absorption. As a result, the conjugated AIEgen-chloroplasts exhibited better capability of water splitting and election separation for adenosine triphosphate (ATP) generation, which are important products in photosynthesis. This is the first AIEgen-based conjugation strategy reported for improving solar-energy utilization and augmenting photosynthetic efficiency.

\section{INTRODUCTION}

The sun directly or indirectly provides solar and thermal energies for almost all living organisms. And the familiar chemical fuels (e.g., coal, petroleum oil, and natural gas) essentially is the conversion and transmission from solar energy as well.(1) Most of the solar energy is converted to chemical energy and stored in green plants by photosynthesis, and all our foods extraordinarily rely on the photosynthesis in origin.(2) So the fact is that the sun supports all life on the earth, and the photosynthesis is the foundation of sustaining planet living. As the discovery and study in 1954, the isolated chloroplast (cp) was found to be the basic light-driven metabolic factory of higher plant cells where carbohydrates and oxygen are produced from carbon dioxide and water. $(3,4)$ The contained chlorophyll A and chlorophyll B are the main photosynthetic pigments of chloroplasts. As the initial step of photochemical reaction, they could harvest the sunlight and activate the subsequent process.(5) However, not all solar energy could be absorbed and utilized by the photosynthetic pigments because of the incomplete match between the solar radiation spectrum and the absorption profile of chloroplasts. The absorbance of the chlorophyll A and chlorophyll B is mainly located at blue and red spectral regions (Fig. 1a), while the solar energy is distributed evenly across the entire spectrum, without any gaps, dips, or spikes.(6) Additionally, the nonvisible ultraviolet region is likely to be a harmful radiation source, resulting in the damage of DNA and protein.(7) To improve the efficacy of photosynthesis, the required effective strategy is that can regulate the natural photosynthetic unit and redistribute the solar energy.

Several materials have been tried to convert the unutilized solar energy into photosynthetically active radiation (PAR).(8-11) Among them, partly natural characteristics of inorganic luminescent materials have shortages of high cost of raw material, difficult operation for purification, and poor. In contrast, organic fluorescent materials could avoid these drawbacks and thus they are more suitable for this domain.(2, 12-14) But some of the hydrophobic fluorescent dyes are apt to present as an accumulation state, and suffer from aggregation-caused quenching (ACQ) effect in aqueous.(15) The excitons of ACQ molecules dissipate their energy through nonradiative decay, rather than 
photoluminescence. Obviously, they are not well-matched materials for the goals of highly efficient solar energy transfer and conversion.

On the contrary, the concept of aggregation-induced emission (AIE) describing an unusual photophysical phenomenon was proposed by Tang et al. in 2001, in which the accumulated molecules could still exhibit strong fluorescence instead of emission quenching.(16) As the defined mechanism of the AIE phenomenon, the aggregates of AIEgens could lead to the restriction of intramolecular motion (RIM) and largely inhibit the nonradiative decay, resulting in the escalation of luminescence.(17) It means that the excitation energy of molecules is more likely to appear as optical energy, rather than other pathways. So AIEgen is a more desirable choice for light-harvesting and solar energy conversion. Inspired by the concept of cellular engineering,(18) chloroplast-based bioconjugation is a proper way to introduce AIEgens into the photosynthetic systems. Just like the manipulation of a live cell, the conjugation strategy toward chloroplast is more beneficial to explore the scientific essence and maximize photosynthetic efficiency. And the double-membrane envelope and complex internal organization of chloroplasts could apply many possible reactive sites for the proposed modification. Considering the requirements of mild reaction condition, facile site-specificity, and the maintained biofunction, the satisfied metal-free bioconjugation reaction between activated alkynes and native amino groups is projected in this strategy. $(19,20)$

In this contribution, we designed two novel AIEgens with activated alkyne groups by the derivatization of tetraphenylethylene (TPE) and triphenylamine (TPA). By conjugation strategy, they were successfully modified on the chloroplasts through the metal-free "Click" reaction based on high reactivity of alkynes to amines (Fig. 1b). Owing to their excellent light-harvesting and energy transfer ability, the synthesized TPE-PPO and TPA-TPO respectively realized the conversion of harmful ultraviolet radiation (HUVR) and photosynthetically inefficient radiation (PIR) into the photosynthesis active radiation (PAR). The AIEgen-chloroplasts bioconjugation effectively improved solarenergy utilization and photosynthetic activity.
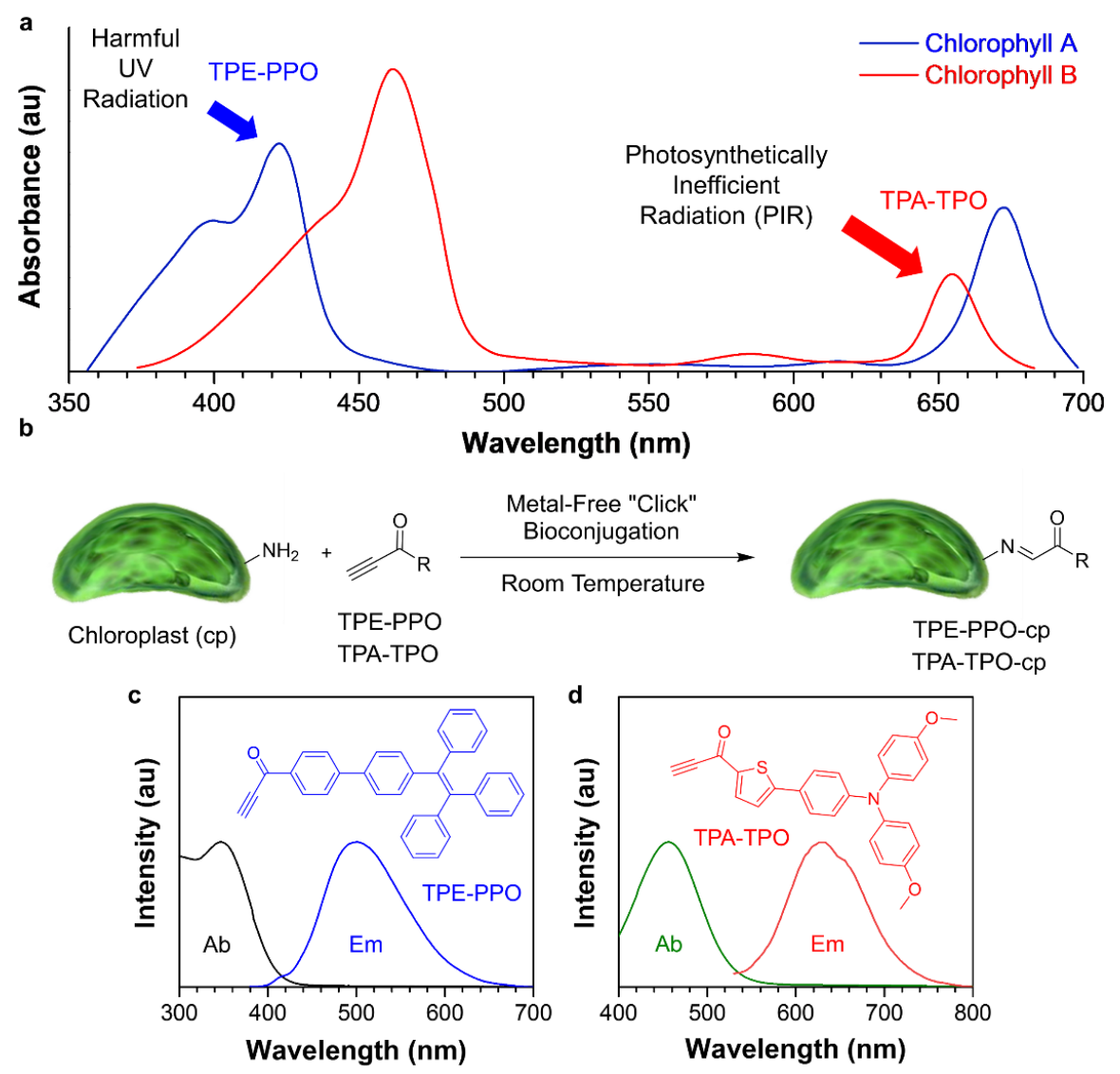

Fig. 1. (a) Diagrammatic illustration of solar energy conversion from harmful ultraviolet radiation (HUVR) and photosynthetically inefficient radiation (PIR) to chlorophylls A and B in chloroplasts (cp) for augmenting photosynthesis. (b) Facile conjugation of AIEgens to cp via metal-free "Click" reaction of activated alkynes and natural amino groups under ambient conditions. $\mathrm{c}$ and d) The absorption (Ab) and emission (Em) spectra of the alkyne-functionalized AIEgens: TPE-PPO (c) and TPA-TPO (d). 


\section{RESULTS AND DISCUSSION}

The photophysical property of the AIEgens was first investigated, which was useful to evaluate the light regulating ability and the possibility of energy transfer. Refer to the absorption spectrum of chloroplasts (Fig. S7), the wavelength of solar energy less than $350 \mathrm{~nm}$ was hard to be captured by the chloroplasts, while the blue and red lights could be efficiently absorbed for photosynthesis. Coincidentally, the absorption range of TPE-PPO was from 300 to $400 \mathrm{~nm}$, and the emission range was from $400 \mathrm{~nm}$ to $650 \mathrm{~nm}$ with blue fluorescence (Fig. 1c). The spectral matching suggested that the TPE-PPO could convert the ineffective HUVR into blue fluorescence for chloroplast absorption. Similarly, the absorption range (around $500 \mathrm{~nm}$ to $680 \mathrm{~nm}$ ) of TPA-TPO belonged to the photosynthetically inefficient radiation (PIR) of solar energy, while the red fluorescence $(540 \mathrm{~nm}$ to $780 \mathrm{~nm}$ ) could be capture by chloroplast (Fig. 1d). And then the titration experiment of AIEgens toward chloroplasts was conducted to further verify the hypothesized idea of energy conversion (Fig. S8). With the addition of the chloroplasts, the emission intensity of AIEgens was decreased gradually. This phenomenon demonstrated that the emission from AIEgens could be efficiently captured by chloroplasts, and the typical energy transmission from AIEgens to chloroplasts was accused. So, the matched optical characteristic indicated that the TPE-PPO and TPA-TPO are expected to intelligently regulate solar light as the plant demand and further promote the solar-energy utilization and photosynthetic effects.

We further investigated their AIE effects and metal-free "Click" reactivity, which are important for the manipulation of chloroplast and the solar-energy capture and utilization. As one of the most distinctive characters, the AIE phenomenon contributed to the outstanding optical performance of AIEgens in the physiological micro-environment. As shown in Fig. 2a, 2b and S9, the AIE effect was conducted in DMSO/water mixtures with different water fractions $\left(f_{\mathrm{w}}\right)$. TPE-PPO and TPA-TPO both exhibited weak fluorescence emission when the water content was less than $40 \%$, while the emission intensity gradually increased when further increasing the water content. The peak of TPE-PPO was at about $515 \mathrm{~nm}$, and that of TPA-TPO was at $630 \mathrm{~nm}$ (Fig. 1c and 1d). It demonstrated that both TPE-PPO and TPA-TPO could form the aggregated state and induce stronger fluorescent signals under the aqueous environment. So, their quantified AIE effect and photophysical property could satisfy our desirable biological application. Besides, the AIE effect was a utility tool to qualitatively analyze the metal-free "Click" reactivity of TPE-PPO and TPA-PPO. The bovine serum albumin (BSA), a single polypeptide and consisted of 59 lysine residues, was treated as the model target for the conjugated reaction. As shown in Fig. 2c, a clear fluorescent signal could be collected from the AIEgensmodified with BSA, while the pristine BSA showed very faint fluorescence. Based on the well-defined AIE mechanism, the linkage between AIEgens and BSA could hinder the inherent intramolecular motion of TPE-PPO and TPA-TPO, inducing the fluorescence emission. In addition, the conjugation efficiency of the AIEgens-modified BSA was further quantified by matrix-assisted laser desorption/ionization (MALDI). The positive shift of mass spectra in Fig. 2d showed that the molecular weight of the modified BSA (more than $67 \mathrm{kDa}$ ) was larger than that of the pristine BSA (around $66 \mathrm{kDa}$ ). The further calculated results demonstrated that about two AIEgen units could link with one BSA molecule, under a moderate reaction condition. The MALDI results attested to the above fluorescence increasing, and it verified the covalent reactivity of TPE-PPO and TPA-TPO again. Because approximately 3000 proteins were involved in the chloroplast and cooperatively worked as the organelle for photosynthesis.(21) It was foreseen that both the TPE-PPO and TPA-TPO could be modified on chloroplasts stably.
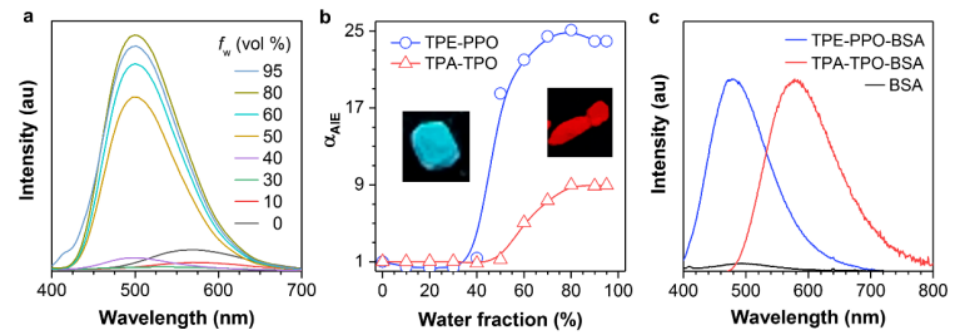

d

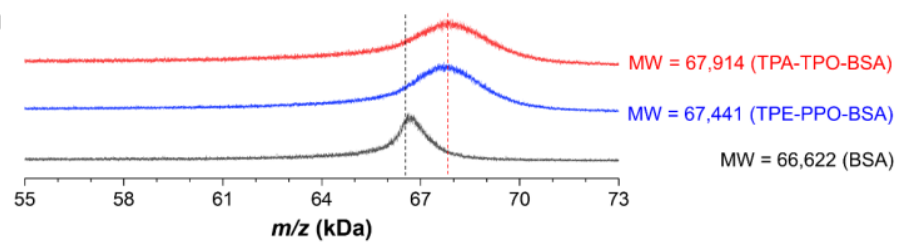

Fig. 2. (a) Fluorescent spectra of TPE-PPO in DMSO/H2O mixtures with different water fractions $\left(f_{\mathrm{w}}\right)$. (b) Plots of AIE $v s . f_{\mathrm{w}}$ of AIEgens: TPEPPO at $505 \mathrm{~nm}$ and TPA-TPO at $603 \mathrm{~nm}$; AIE $=I / I_{0}$. Insets: fluorescent images of solid powders of PTE-PPO (left) and TPA-TPO (right) taken 
under $365 \mathrm{~nm}$ illumination. (c) Fluorescent spectra of pristine and AIEgen-conjugated bovine serum albumins (BSA). (d) Laser desorption mass spectra of BSA and its AIEgen conjugates: TPE-PPO-BSA and TPA-TPO-BSA.

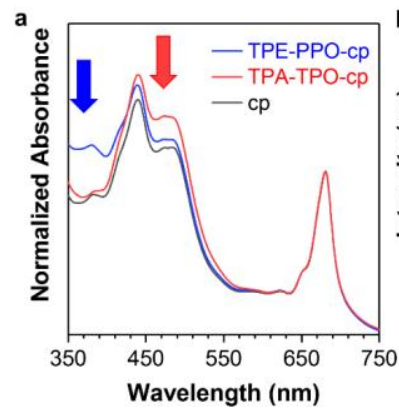

c AlEgen
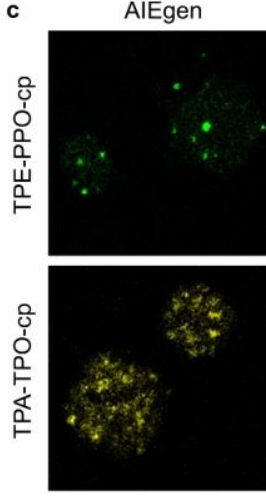

Fig. 3. (a) Absorption spectra of pristine and AIEgen-cp normalized at $680 \mathrm{~nm}$. (b) Fluorescence spectra of cp and AIEgen-cp. (c) Confocal images of TPE-PPO-cp, TPA-TPO-cp, and cp. Pseudo green color is from TPE-PPO ( $\lambda$ ex $=405 \mathrm{~nm}, \lambda \mathrm{em}=450-550 \mathrm{~nm})$; Pseudo yellow color is from TPATPO $(\lambda \mathrm{ex}=405 \mathrm{~nm}, \lambda \mathrm{em}=500-620 \mathrm{~nm})$, Pseudo red color is from chloroplasts $(\lambda \mathrm{ex}=488 \mathrm{~nm}, \lambda \mathrm{em}=680-750 \mathrm{~nm})$.

Subsequently, spectroscopy and confocal laser scanning microscopy (CLSM) were used to characterize the conjugated behavior between AIEgens and chloroplasts. As shown in Fig. 3a and 3b, the characteristic absorption peak at $360 \mathrm{~nm}$ and emission peak at $500 \mathrm{~nm}$ of AIEgen-chloroplasts verified that the TPE-PPO has been conjugated to chloroplasts facilely. Similarly, the absorbance peak at $460 \mathrm{~nm}$ and the emission range around $600 \mathrm{~nm}$ demonstrated that the TPA-TPO could do that as well. Relying on the photophysical information, fluorescent microimaging was applied to provide more direct evidence about the conjugation. As shown in Fig. 3c and Fig. S10, the fluorescence signal from TPE-PPO and TPA-TPO (pseudo green color and yellow color) were only appeared and well collected in the AIEgen-chloroplasts. Moreover, these spots partly overlapped and colocalized with the autofluorescence of chloroplasts (pseudo red color). These visual results further presented the conjugation effects more credibly. Furthermore, the time-dependent experiments demonstrated that the reaction process was highly effective and accrued within 20 mins (Fig. S11). All the above results confirmed that the AIEgen-chloroplasts had been constructed by a facile conjugation strategy.

The above hypothesis about the improved photosynthetic activity was measured by 2,6-dichlorophenolindophenol (DCPIP). As an artificial electron acceptor, the DCPIP could trap the electrons generated from the process of water splitting and electron separation in photosystem II (Fig. 4a). So the reduction rate of DCPIP could evaluate the photosynthetic activity of AIEgen-chloroplasts. Following this guild, the decrease of DCPIP absorbance at $600 \mathrm{~nm}$ by AIEgen-chloroplasts was measured and calculated. The optimum state was measured by linking different amounts of AIEgens (from $1 \mu \mathrm{M}$ to $4 \mu \mathrm{M}$ ) with $20 \mathrm{mg} / \mathrm{mL}$ chloroplasts. Next, they were separately conducted under the irradiation of $0.5 \mathrm{~mW} / \mathrm{cm}^{2}$ UV light and $1 \mathrm{~mW} / \mathrm{cm}^{2}$ visual light. As shown in Fig. S12, all the chloroplast modified with AIEgens displayed a higher reductive ability toward DCPIP comparing with the pristine chloroplasts, which indicated that rational conjugation had fewer effects on the bioactivity of chloroplasts. Moreover, the $2 \mu \mathrm{M}$ TPE-PPO and $1 \mu \mathrm{M}$ TPA-TPO could make the chloroplasts reached the optimum test condition. The roles of light-harvesting and energy transfer of AIEgens was further verified by changing irradiation intensity. Comparing with the pristine chloroplasts, the TPE-PPO modified ones could result in the absorbance of DCPIP decreasing more rapidly with prolonged irradiation. And the gap between them could be further widened when the UV light intensity was increased from 0.5 $\mathrm{mW} / \mathrm{cm}^{2}$ to $1 \mathrm{~mW} / \mathrm{cm}^{2}$ (Fig. 2b). It was because that the TPE-PPO-chloroplasts generated more electrons from water 
separation and distinctly accelerated the reduction rate of DCPIP under irradiation. And the stronger light could further promote the photosynthetic reaction rate. Similarly, the chloroplasts modified with TPA-TPO presented a promoted tendency under visual light irradiation. These results testified that the light conversion effects of TPE-PPO and TPATPO contributed to the improved photosynthesis by applying more energy for chloroplasts absorption. As another important product of the photosynthetic system, the adenosine triphosphate (ATP) was investigated and measured by the luciferin/luciferase assay in addition. The results demonstrated that both the UV and visual irradiation could drive the AIEgen-chloroplasts to generate more ATP (Fig. 4c). The increased yield of ATP was consistent with the electron separation results of DCPIP assay. The mechanism of the optimized light-harvesting ability was further confirmed by chlorophyll fluorescence parameters. The results showed that the AIEgens could improve the light absorption capability, energy trapping, and energy efficiency for electron transfer of per reaction center of the chloroplasts (Table S1). So the more efficient solar energy utilization can be described as the promoted light-harvesting and energy transfer. The bioconjugation strategy based on TPE-PPO and TPA-TPO can noticeably augment the photosynthesis of chloroplasts.
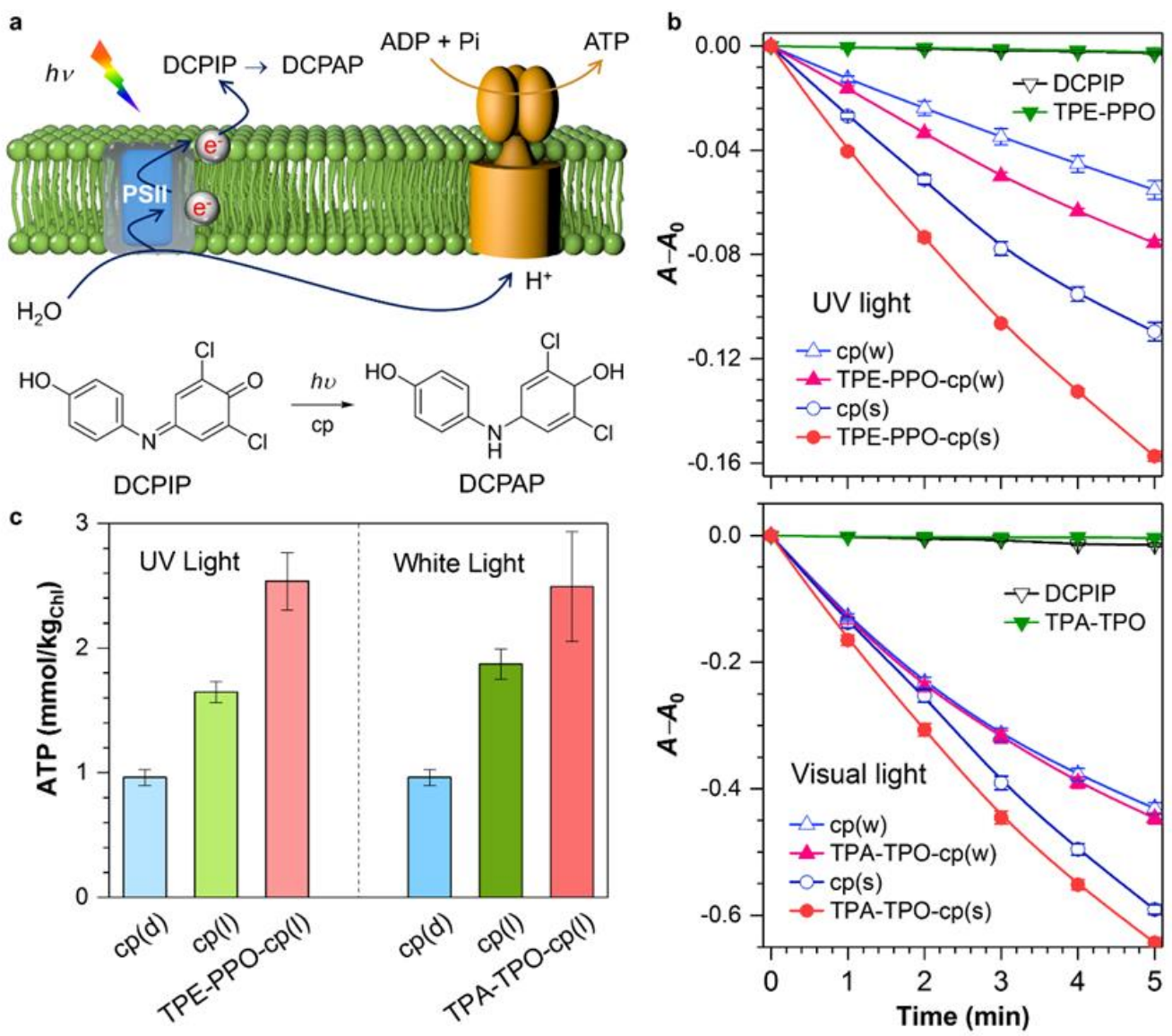

Fig. 4. (a) Simplified illustration of photoinduced electron generation (PEG) in the photosynthesis process of cp; reaction schedule for evaluating PEG efficiency by 2,6-dichlorophenolindophenol (DCPIP). b) Time dependence of absorbance of DCPIP at $600 \mathrm{~nm}$ in aqueous suspensions of pristine and AIEgen-cp under irradiation of (upper panel) UV light of 0.5 (weak, w) and $1 \mathrm{~mW} / \mathrm{cm}^{2}$ (strong, s]; (lower panel) visual light of 1 (weak, w) and $2 \mathrm{~mW} / \mathrm{cm}^{2}$ (strong, s). c) Production of adenosine triphosphate (ATP) in pristine and AIEgen-cp in the dark (d) and under light irradiation (1)

\section{CONCLUSION}

In summary, we synthesized two new AIEgens with the activated alkyne units, and further artificially conjugated them on chloroplasts by metal-free "Click" reaction at a mild condition. For the reconstructive photosynthetic system, due to their outstanding light-harvesting and energy transfer characteristics, the ultraviolet and green light irradiations could be respectively absorbed, and converted into blue light and red light for the photosynthesis of chloroplasts. In this way, the AIEgens realized the conversion of harmful ultraviolet radiation (HUVR) and photosynthetically inefficient radiation (PIR) to the photosynthesis active radiation (PAR) for more effective photosynthetic reactions. 
In this proof-of-concept study, the constructed AIEgen-chloroplasts with enhanced light-harvesting ability owned better capability of water splitting and electron separation for more ATP production. The facile conjugation strategy could efficiently improve solar energy utilization and augment the photosynthetic efficiency. Furthermore, this work exhibited that the excellent optical functional materials had enormous potential to develop modern bioenergy in the future. The material chemists have a great stage for reforming the chloroplasts, optimizing the photosynthetic system, and transforming nature.

\section{REFERENCES AND NOTES}

1. T. Vlachogianni and A. Valavanidis, Energy and environmental impact on the biosphere energy flow, storage and conversion in human civilization. Am. Educ. Res. J. 3, 68-78 (2013).

2. X. Zhou, Y. Zeng, Y. Tang, Y. Huang, F. Lv, L. Liu, S. Wang, Artificial regulation of state transition for augmenting plant photosynthesis using synthetic light-harvesting polymer materials. Sci. Adv. 6, eabc5237 (2020).

3. D. I. Arnon, M. B. Allen, F. R. Whatley, Photosynthesis by isolated chloroplasts. Nature. 174, 394-396 (1954).

4. H. Kirchhoff, Chloroplast ultrastructure in plants. New Phytologist. 223, 565-574 (2019).

5. M. R. Wasielewski, Self-assembly strategies for integrating light harvesting and charge separation in artificial photosynthetic systems. Acc. Chem. Res. 42, 1910-1921 (2009).

6. J. A. Herron, J. Kim, A. A. Upadhye, G. W. Huber, C. T. Maravelias, A general framework for the assessment of solar fuel technologies. Energy Environ. Sci. 8, 126-157 (2014).

7. A. Stapleton, Ultraviolet radiation and plants: burning questions. Plant Cell. 4, 1353-1358 (1992),

8. D. Li, W. Li, H. Zhang, X. Zhang, J. Zhuang, Y. Liu, C. Hu, B. Lei, Far-red carbon dots as efficient light-harvesting agents for enhanced photosynthesis. ACS Appl. Mater. Interfaces. 12, 21009-21019 (2020).

9. Y. Xu, J. Fei, G. Li, T. Yuan, X. Xu, C. Wang, J. Li, Optically Matched Semiconductor Quantum Dots Improve Photophosphorylation Performed by Chloroplasts. Angew. Chem. Int. Ed. 130, 6642-6645 (2018).

10. X. Xu, W. Li, C. Hu, B. Lei, X. Zhang, Y. Li, Q. Zhan, Y. Liu, J. Zhuang, Promoting the growth of mung bean plants through uptake and light conversion of NaYF4:Yb,Er@CDs nanocomposites. ACS Sustainable Chem. Eng. 8, 9751-9762 (2020).

11. Y. Zeng, X. Zhou, R. Qi, N. Dai, X. Fu, H. Zhao, K. Peng, H. Yuan, Y. Huang, F. Lv, L. Liu, S. Wang, Photoactive conjugated polymer-based hybrid biosystems for enhancing cyanobacterial photosynthesis and regulating redox state of protein. Adv. Funct. Mater. n/a, 2007814.

12. Y. Wang, S. Li, L. Liu, F. Lv, S. Wang, Conjugated polymer nanoparticles to augment photosynthesis of chloroplasts. Angew. Chem. Int. Ed. 56, 5308-5311 (2017).

13. Z. Wang, D. Gao, Y. Zhan, C. Xing, Enhancing the light coverage of photosynthetic bacteria to augment photosynthesis by conjugated polymer nanoparticles. ACS Appl. Bio Mater. 3, 3423-3429 (2020).

14. Z. Li, H. Bai, S. Jia, H. Yuan, L. H. Gao, H. Liang, Mater. Chem. Front. doi:10.1039/D0QM00837K (2020).

15. Z. Zhao, H. Zhang, J. W. Y. Lam, B. Z. Tang, Aggregation-induced emission: new vistas at the aggregate level. Angew. Chem. Int. Ed. 59, 9888-9907 (2020).

16. J. Luo, Z. Xie, J. W. Y. Lam, L. Cheng, B. Z. Tang, H. Chen, C. Qiu, H. S. Kwok, X. Zhan, Y. Liu, D. Zhu, Aggregation-induced emission of 1-methyl-1,2,3,4,5-pentaphenylsilole. Chem. Commun. 1740-1741 (2001).

17. R. Hu, A. Qin, B. Z. Tang, AIE polymers: synthesis and applications. Prog. Polym. Sci. 100, 101176 (2020).

18. M. Li, C. Nie, L. Feng, H. Yuan, L. Liu, F. Lv, S. Wang, Conjugated Polymer nanoparticles for cell membrane imaging. Chem. Asian J. 9, 3121-3124 (2014).

19. X. Hu, X. Zhao, B. He, Z. Zhao, Z. Zheng, P. Zhang, X. Shi, R. T. K. Kwok, J. W. Y. Lam, A. Qin, B. Z. Tang, A simple approach to bioconjugation at diverse levels: metal-free click reactions of activated alkynes with native groups of biotargets without prefunctionalization. Research. 2018, 1-12 (2018).

20. R. Hu, X. Chen, T. Zhou, H. Si, B. He, R. T. K. Kwok, A. Qin, B. Z. Tang, Lab-in-cell based on spontaneous amino-yne click polymerization. Sci. China Chem. 62, 1198-1203 (2019).

21. P. Jarvis, Targeting of nucleus-encoded proteins to chloroplasts in plants. New Phytologist. 179, 257-285 (2008).

Acknowledgments: This work is supported by the National Natural Science Foundation of China (21788102), the Research Grants Council of Hong Kong (N-HKUST609/19, 1606620 and C6009-17G), the Innovation and Technology Commission (ITC-CNERC14SC01), the National Key Research and Development program of China (2018YFE0190200), and the Science and Technology Plan of Shenzhen (JCYJ20180507183832744)

Competing interests: The authors declare no conflict of interest. Data and materials availability: All data needed to evaluate the conclusions in the paper are present in the paper and/or the Supplementary Materials. Additional data related to this paper may be requested from the authors. 